\title{
New Bioactive Calcium Silicate Cement Mineral Trioxide Aggregate Repair High Plasticity (MTA HP) - A Systematic Review
}

\author{
Mirona Palczewska-Komsa, Kinga Kaczor-Wiankowska * (D) and Alicja Nowicka (D)
}

Department of Conservative Dentistry and Endodontics, Pomeranian Medical University in Szczecin, Powstancow Wielkopolskich 72, 70-111 Szczecin, Poland; mpalczewskakomsa@op.pl (M.P.-K.); nowicka6@gmail.com (A.N.)

* Correspondence: kaczorkinga@gmail.com

Citation: Palczewska-Komsa, M.; Kaczor-Wiankowska, K.; Nowicka, A. New Bioactive Calcium Silicate Cement Mineral Trioxide Aggregate Repair High Plasticity (MTA HP) —A Systematic Review. Materials 2021, 14 , 4573. https://doi.org/10.3390/ ma14164573

Academic Editor: Edgar Schäfer

Received: 7 July 2021

Accepted: 12 August 2021

Published: 14 August 2021

Publisher's Note: MDPI stays neutral with regard to jurisdictional claims in published maps and institutional affiliations.

Copyright: (c) 2021 by the authors. Licensee MDPI, Basel, Switzerland. This article is an open access article distributed under the terms and conditions of the Creative Commons Attribution (CC BY) license (https:/ / creativecommons.org/licenses/by/ $4.0 /)$.
Abstract: Bioactive calcium silicate cement Mineral Trioxide Aggregate (MTA) has been used for years as a gold standard in intravital pulp treatment and specialist endodontic procedures. Owing to flaws of the material, the manufacturers have been trying to enhance and produce materials showing improved physical, chemical and biological parameters. One of the new calcium-silicate cements based on mineral trioxide aggregate, however without some flaws exhibited by the cement, is Mineral Trioxide Aggregate Repair High Plasticity (MTA HP). The aim of the present paper was a systematic literature review concerning the MTA HP material used nowadays in dentistry, as a review of its specific features. The present paper is the first article providing a systematic literature review on MTA HP. The aim of the present article is the better understanding of MTA HP properties, which can aid the decision-making process in endodontic treatment.

Keywords: mineral trioxide aggregate; MTA repair HP; bioactive calcium-silicate cement; review

\section{Introduction}

Bioactive materials have been successfully used in dentistry, particularly in endodontic procedures. The bioceramics applied in the treatment are classified as bioinert, bioactive and biodegradable materials [1-4]. The shared feature of the ceramic materials is their role in minimally invasive endodontics and regenerative endodontics. Moreover, these materials can be used for root canal sealing and root cavity filling [1].

The leading bioceramic material that has been used as a gold standard in biological treatment of the pulp and specialist endodontic procedures is Mineral Trioxide Aggregate MTA [5,6]. MTA is available in two variations, depending on the color, i.e., gray and white MTA. The major difference between gray MTA (ProRoot MTA, Densply Sirona) and white MTA (White ProRoot MTA, Densply Sirona) is the concentrations of $\mathrm{Al}_{2} \mathrm{O}_{3}, \mathrm{MgO}$ and $\mathrm{FeO}[7,8]$. The traditional MTA compositions have some drawbacks: discoloration of the tooth and marginal gums, long setting time, and difficult handling [6,9]. The difficult handling of MTA seems to be aggravated in such procedures as root, furcation perforation or filling of root-end cavities. [5,10]. Recently, new formulations have been introduced [6]. Among them, MTA HP, (Angelus, Londrina, PR, Brazil) has been proposed. According to the manufacturer, it is an endodontic restorative cement of high-plasticity consisting of mineral oxides in the form of fine hydrophilic particles. The formula of MTA HP maintains all the chemical and biological characteristics of the original MTA preparation. Table 1 shows the composition of MTA (ProRoot MTA, Densply Sirona) cement used as a gold standard in endodontics and the properties of MTA HP material under study [11-13].

MTA HP is indicated for the following: iatrogenic and carious furcation the treatment of root canal perforation; periapical surgery with retrograde root canal filling; the root canal treatment of root perforation due to internal resorption; pulp capping; pulpotomy 
(the removal of the affected pulp from the crown of the tooth while maintaining viability and function of the pulp) [14]. The present paper is the first to provide a systematic literature review concerning MTA HP material. The aim of the study was to analyze the physicochemical and biological properties in in vitro and in vivo research with the use of MTA HP material. This will improve better understanding of MTA HP properties which can aid the decision-making process in endodontic treatment.

Table 1. Detailed manufacturer data on ProRoot MTA and MTA repair HP.

\begin{tabular}{|c|c|c|c|}
\hline Material & Producer & Composition & Properties \\
\hline Pro Root MTA & $\begin{array}{l}\text { Dentsply Sirona, } \\
\text { Tulsa, USA }\end{array}$ & $\begin{array}{c}\text { Powder: tricalcium silicate } \\
(\mathrm{CaO})_{3} \cdot \mathrm{SiO}_{2} \\
\text { dicalcium silicate }(\mathrm{CaO})_{2} \cdot \mathrm{SiO}_{2} \\
\text { tricalcium aluminate }(\mathrm{CaO})_{3} \cdot \mathrm{Al}_{2} \mathrm{O}_{3} \\
\text { bismuth oxide } \mathrm{Bi}_{2} \mathrm{O}_{3} \\
\text { gypsum } \mathrm{CaSO}_{4} \cdot 2 \mathrm{H}_{2} \mathrm{O} \\
\text { Liquid: distilled water } \mathrm{H}_{2} \mathrm{O}\end{array}$ & $\begin{array}{l}\text { The handling can be difficult, } \\
\text { the initial setting time is long ( } 78 \mathrm{~min}) \text {, } \\
\text { the use in the visible crown area may lead to } \\
\text { tooth discoloration, } \\
\text { the lowest radiopacity of all MTA materials }\end{array}$ \\
\hline MTA Repair HP & $\begin{array}{c}\text { Angelus, Londrina, } \\
\text { Brasil }\end{array}$ & $\begin{array}{c}\text { Powder: Tricalcium Silicate } \\
\text { 3CaO· } \mathrm{SiO}_{2} \\
\text { Dicalcium Silicate } 2 \mathrm{CaO} \cdot \mathrm{SiO}_{2}, \\
\text { Tricalcium Aluminate } 3 \mathrm{CaO} \cdot \mathrm{Al}_{2} \mathrm{O}_{3}, \\
\text { Calcium Oxide } \mathrm{CaO}, \\
\text { Calcium Tungstate } \mathrm{CAWO} \\
\text { Liquid: Water and Plasticizer }\end{array}$ & $\begin{array}{l}\text { Material solidifies when kept in a wet } \\
\text { environment after spatulation, } \\
\text { the initial setting time is approximately } 15 \text { min; } \\
\text { absence of dental discoloration due to the } \\
\qquad \mathrm{CaWO}_{4} \text { radiopacifier used. } \\
\text { radiopacity: nearly matches that of gutta-percha } \\
\text { More radiopaque thanPro Root MTA; }\end{array}$ \\
\hline
\end{tabular}

\section{Material and Methods}

\subsection{Review Questions}

The review of the literature was based on the Preferred Reporting Items for Systematic Reviews (PRISMA) (Figure 1) [15]. This article aims to answer the following questions:

1. Which biological, physical and chemical parameters of MTA HP influence its therapeutic efficacy?

2. What are the disadvantages and advantages of MTA HP in comparison with other calcium silicate cements?

\subsection{Information Sources and Search Strategy}

Five databases (PubMed/MEDLINE, Web of Science, Scopus, Dentistry and Oral Sciences Source (EBSCOhost, Wiley Online Library and Science Direct) were searched electronically by two independent reviewers (M.PK and K.KW) for publications involving information about MTA HP. Any disagreements were resolved by mutual discussion and consensus with an experienced researcher (A.N.). Publications were searched without a year limit. The last search was conducted on 22 May 2021. The search phrases are presented in Table 2. All papers were imported into the Mendeley (2020 Mendeley Ltd., Elsevier) program for the management of scientific bibliography. Having removed the duplicates, all articles (titles, abstracts) were examined. Articles were extracted based on the inclusion criteria listed below:

1. In vitro and in vivo studies that evaluated MTA HP.

2. Publications concerning the physicochemical and biological properties of the MTA HP.

\subsection{Eligibility Criteria}

The research included in vitro and in vivo studies that compared the clinical effectiveness of MTA HP with different bioceramic materials. The author excluded articles of which the full texts were not available in English. All exclusion and inclusion criteria are described in Table 3. Data concerning the papers, properties, application material, the number of cases/controls, the type of clinician problem, observation time, retention, marginal adaptation and solubility were extracted from the selected papers by means of 
a standardized sheet in Microsoft Office Word 2010 (Microsoft Corporation, Redmond, WA, USA).

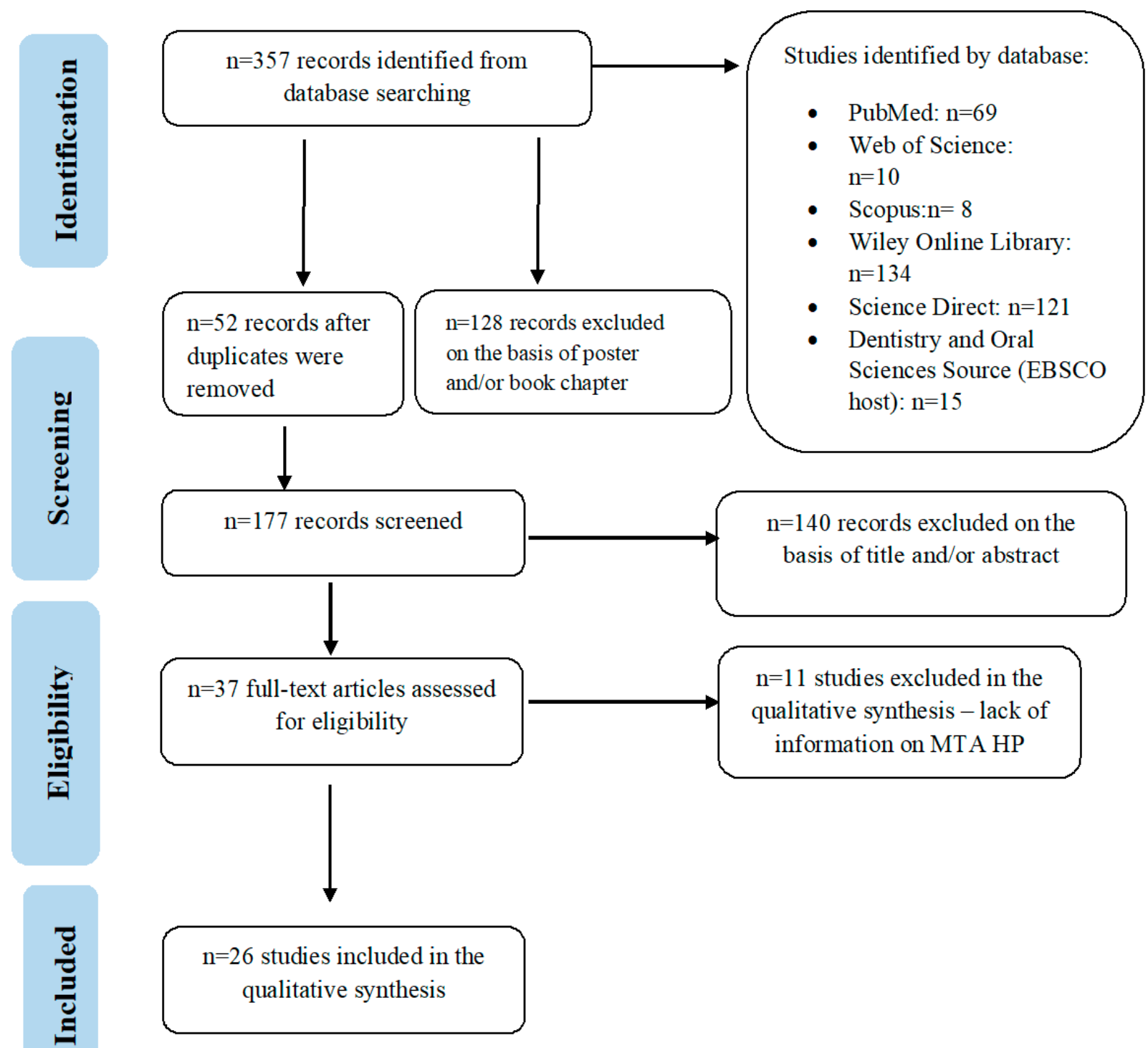

Figure 1. Search flow diagram compliant with PRISMA guidelines. 
Table 2. The search phrases.

\begin{tabular}{|c|c|c|c|}
\hline Database & Filtres & Number of Phrase & Search Phrases \\
\hline \multirow{4}{*}{$\begin{array}{l}\text { Medline } \\
\text { (PubMed) } \\
\quad(69)\end{array}$} & $\begin{array}{c}\text { Abstract } \\
\text { Free full text } \\
\text { Full text } \\
\text { Clinical Trial } \\
\text { Journal Article } \\
\text { Randomized } \\
\text { Controlled Trial }\end{array}$ & 1 & (teeth) OR (tooth) Filters: Abstract, Free full text, Full text, Clinical Trial, Journal Article, Randomized Controlled Trial \\
\hline & - & 2 & $\begin{array}{l}\text { ((((MTA HP[Title/Abstract]) OR (MTA high repair[Title/Abstract])) OR (MTA high plasticity[Title/Abstract])) OR } \\
\text { (MTA repair HP[Title/Abstract]) AND ((ffrft[Filter]) AND (fha[Filter]) AND (clinicaltrial[Filter] OR journalarticle[Filter] } \\
\text { OR randomizedcontrolledtrial[Filter]) AND (fft[Filter]) OR (Calcium Silicate-Based))))) Filters: Abstract, Free full text, } \\
\text { Full text, Clinical Trial, Journal Article, Randomized Controlled Trial }\end{array}$ \\
\hline & - & 3 & $\begin{array}{l}\text { (solubility) OR (Ca2+ ions release)) OR (Bond strength)) OR (Film thickness)) OR (Adhesive)) OR (discoloration)) OR } \\
\text { (Radiopacity)) OR (Setting time)) OR (Inflammatory response)) OR (Cytotoxicity)) OR (Apoptosis)) OR (Mineralization)) } \\
\text { OR (Antimicrobal effect)) OR (physicochemical properties)) OR (chemical properties)) OR (biological properities)) OR } \\
\text { (properities) Filters: Abstract, Free full text, Full text, Clinical Trial, Journal Article, Randomized Controlled Trial }\end{array}$ \\
\hline & - & 1 AND 2 AND 3 & $\begin{array}{l}\text { (teeth) OR (tooth) AND(MTA HP[Title/Abstract]) OR (MTA high repair[Title/Abstract]) OR (MTA high } \\
\text { plasticity[Title/Abstract])) OR (MTA repair HP[Title/Abstract]) OR (Calcium Silicate-Based) AND (solubility) OR (Ca2+ } \\
\text { ions release)) OR (Bond strength)) OR (Film thickness)) OR (Adhesive) OR (discoloration)) OR (Radiopacity)) OR } \\
\text { (Setting time)) OR (Inflammatory response) OR (Cytotoxicity)) OR (Apoptosis)) OR (Mineralization)) OR (Antimicrobal } \\
\text { effect) OR (physicochemical properties) OR (chemical properties)) OR (biological properties) OR (properties) }\end{array}$ \\
\hline $\begin{array}{l}\text { Web of } \\
\text { Science } \\
(10)\end{array}$ & $\begin{array}{c}\text { Web of Science } \\
\text { Categories: } \\
\text { Dentistry } \\
\text { Oral } \\
\text { Surgery } \\
\text { Medicine Document } \\
\text { Types: } \\
\text { Article }\end{array}$ & 1 & TOPIC: (teeth) OR TOPIC: (tooth) \\
\hline- & - & 2 & $(\mathrm{TS}=($ MTA HP OR MTA high plasticity OR MTA repair HP) \\
\hline- & - & 3 & $\begin{array}{l}\text { (TS = (solubility OR Ca2+ ions release OR Bond strength OR Film thickness OR Adhesive OR discoloration OR } \\
\text { Radiopacity OR Setting time OR Inflammatory response OR Cytotoxicity OR Apoptosis OR Mineralization OR } \\
\text { Antimicrobal effect OR physicochemical properties OR chemical properties OR biological properties OR properties) }\end{array}$ \\
\hline
\end{tabular}


Table 2. Cont.

\begin{tabular}{|c|c|c|c|}
\hline Database & Filtres & Number of Phrase & Search Phrases \\
\hline- & - & 1 AND 2 AND 3 & $\begin{array}{l}\text { (TOPIC: (teeth) OR TOPIC: (tooth) AND (TS = (MTA HP OR MTA high plasticity OR MTA repair HP) AND (TS = } \\
\text { (solubility OR Ca2+ ions release OR Bond strength OR Film thickness OR Adhesive OR discoloration OR Radiopacity } \\
\text { OR Setting time OR Inflammatory response OR Cytotoxicity OR Apoptosis OR Mineralization OR Antimicrobal effect } \\
\text { OR physicochemical properties OR chemical properties OR biological properties OR properties) }\end{array}$ \\
\hline $\begin{array}{c}\text { Scopus } \\
(8]) \\
\text { Wiley Online } \\
\text { Library } \\
(134)\end{array}$ & $\begin{array}{l}\text { Language: } \\
\text { English } \\
\text { Subjects: } \\
\text { Dentistry } \\
\text { Journals } \\
\text { Subjects: } \\
\text { Dentistry }\end{array}$ & 1 & teeth OR tooth \\
\hline- & - & 2 & MTA HP OR MTA high plasticity OR MTA repair HP \\
\hline- & - & 3 & $\begin{array}{l}\text { solubility OR ca2+ AND ions AND release OR bond AND strength OR film AND thickness OR adhesive OR } \\
\text { discoloration OR radiopacity OR setting AND time OR inflammatory AND response OR cytotoxicity OR apoptosis OR } \\
\text { mineralization OR antimicrobial AND effect OR physicochemical AND properties OR chemical AND properties OR } \\
\text { biological AND properties OR properties }\end{array}$ \\
\hline $\begin{array}{c}\text { Science Direct } \\
\qquad(121) \\
\text { Dentistry and } \\
\text { Oral Sciences } \\
\text { Source (15) }\end{array}$ & $\begin{array}{l}\text { Subject areas: } \\
\text { Medicine } \\
\text { and Dentistry } \\
\text { Article type: } \\
\text { Research articles } \\
\text { Language: } \\
\text { English }\end{array}$ & 1 & Teeth OR Tooth \\
\hline- & - & 2 & MTA HP OR MTA high plasticity OR MTA repair HP \\
\hline- & - & 3 & Properties \\
\hline- & - & 1 AND 2 AND 3 & (Teeth OR Tooth) AND (properties) AND (MTA HP OR MTA high plasticity OR MTA repair HP) \\
\hline
\end{tabular}


Table 3. Exclusion and inclusion criteria.

\begin{tabular}{ccc}
\hline Criteria & Included & Excluded \\
\hline Full text & Available & Unavailable \\
\hline Publication language & English & Other \\
\hline Type of publication & Journal article & Books, documents \\
\cline { 2 - 3 } & Clinical trail & \\
\cline { 2 - 3 } Type of research & Randomized controlled & \\
\cline { 2 - 3 } & Research articles & \\
\cline { 2 - 3 } & Review & \\
\cline { 2 - 3 } & Meta-analysis & \\
\hline Subject area & Case Report & Other \\
& Dentistry and oral surgery & \\
\hline Publication stage & medicine & \\
\hline
\end{tabular}

\section{Results}

\subsection{Quality Assessment}

The quality of in vitro and in vivo studies was assessed using a modified methodological index for non-randomized studies (MINORS) modified by the authors [16]. In the MINORS scale, the following were taken into account: clear aim, clear application protocol of HP MTA, inclusion of additional patients or animals, prospective data collection, justification of the sample size, observation period, endpoints relevant to the study purpose, blind analysis, adequate control group, contemporary groups, baseline group equivalence and adequate statistical analyzes. The items were rated: 0 , not reported; 1 , introduced but inadequate; and 2, introduced and appropriate. All twelve items were used to evaluate the in vitro studies, while the first eight items were assessed for in vivo studies. The ideal result for comparative studies is 24 , and for non-comparative studies 16 [16]. Test quality classifications were rated as: poor (0-5), good (6-10) and good (11-16) for in vivo tests, and poor (0-8), good (9-16) and good (17-24) for in vitro studies [17]. The results for each item, the overall result and the quality of the study are presented in Tables 4 and 5 for the in vivo and in vitro studies, respectively.

\subsection{Description of the Results}

The database search produced 357 records in total. However, it must be emphasized that not all databases allowed the application of all the inclusion and exclusion criteria, therefore, at a subsequent phase of selection, the research papers not meeting the criteria were excluded by hand and the duplicates were eliminated. Additionally, owing to lack of information by the manufacturer concerning the time since when the product has been commercially available, the database search was not narrowed to particular time period. The results were thoroughly selected with respect to inclusion criteria and 26 research papers were eventually qualified to the analysis. Next, the research papers were divided according to the discussed parameters of MTA HP. 
Table 4. Risk of bias according to the MINORS scale in vivo studies (modified methodological index for nonrandomized studies).

\begin{tabular}{|c|c|c|c|c|c|c|c|}
\hline Source & Ferreira et al., 2019 [6] & El Reash et al., 2019 [9] & Cosme-Silva et al., 2019 [18] & Delfino et al., 2021 [19] & Benetti et al., 2018 [20] & Benetti et al., 2019 [21] & Macedo et al., 2020 [22] \\
\hline Clear aim & 2 & 2 & 2 & 2 & 1 & 1 & 2 \\
\hline Clear MTA HP application protocol & 2 & 2 & 2 & 2 & 2 & 2 & 2 \\
\hline $\begin{array}{l}\text { Inclusion of consecutive } \\
\text { patients, animals }\end{array}$ & 2 & 2 & 2 & 2 & 2 & 2 & 2 \\
\hline Collection of data & 2 & 2 & 2 & 2 & 2 & 2 & 2 \\
\hline Justification of sample size & 0 & 0 & 0 & 0 & 0 & 0 & 0 \\
\hline $\begin{array}{l}\text { Follow-up period appropriate to the aim } \\
\text { of the study }\end{array}$ & 2 & 2 & 2 & 2 & 1 & 1 & 2 \\
\hline Blinded analysis & 0 & 0 & 0 & 0 & 0 & 0 & 0 \\
\hline Study quality & good & good & good & good & fair & fair & good \\
\hline
\end{tabular}

Numbers coding: 2 , introduced and adequate; 1 , introduced but inadequate; 0 , not reported.

Table 5. Risk of bias according to the MINORS scale in vitro studies (modified methodological index for nonrandomized studies).

\begin{tabular}{|c|c|c|c|c|c|c|c|c|}
\hline Source & Barczak et al., 2021 [12] & Benetti et al., 2019 [21] & Tomás-Catalá et al., 2017 [23] & Tomás-Catalá et al., 2018 [24] & Queiroz et al., 2021 [25] & Collado-Gonzlez et al., 2016 [26] & El Reash et al., 2019 [27] & El Reash et al., 2021 [28] \\
\hline Clear aim & 2 & 1 & 2 & 2 & 2 & 2 & 1 & 2 \\
\hline Clear MTA HP application protocol & 2 & 2 & 2 & 2 & 2 & 2 & 2 & 2 \\
\hline Inclusion of consecutive patients, animals & 2 & 2 & 2 & 2 & 2 & 2 & 2 & 2 \\
\hline Collection of data & 2 & 2 & 2 & 2 & 2 & 2 & 2 & 2 \\
\hline Justification of sample size & 0 & 0 & 0 & 0 & 0 & 0 & 0 & 0 \\
\hline Follow-up period appropriate to the aim of the study & 2 & 1 & 0 & 2 & 2 & 2 & 1 & 1 \\
\hline Endpoints appropriate to the aim of the study & 2 & 2 & 2 & 2 & 2 & 2 & 2 & 2 \\
\hline Blinded analysis & 0 & 0 & 0 & 0 & 0 & 0 & 0 & 0 \\
\hline An adequate control group & 2 & 2 & 0 & 0 & 2 & 2 & 2 & 2 \\
\hline Contemporary groups & 2 & 2 & 0 & 0 & 2 & 2 & 2 & 2 \\
\hline Baseline equivalence of groups & 2 & 2 & 0 & 0 & 2 & 2 & 2 & 2 \\
\hline Adequate statistical analyses & 2 & 2 & 2 & 2 & 2 & 2 & 2 & 2 \\
\hline Study quality & good & good & fair & fair & good & good & good & good \\
\hline
\end{tabular}

Numbers coding: 2 , introduced and adequate; 1 , introduced but inadequate; 0 , not reported. 
A total of 16 papers were included into the group discussing the physicochemical parameters of MTA HP [5,16,17,23,29-39]. The analysed selected physicochemical parameters of MTA HP, together with the most important results are given in Table 6.

The group of research papers on the biological properties comprised a total of $18 \mathrm{sci}-$ entific articles, including: seven studies on human cells in vitro [12,23-28], seven on Wistar rats in vivo [6,9,18-22], two research papers on mice in vitro [21,29] and three papers cells in vitro [37-39]. A brief description of the aforementioned studies is presented in Table 7. However, it should be noted that for the purpose of clarity, the table presents only the maximum time of the in vitro and in vivo studies. It should be emphasized that some authors discussed both the physical as well as biological properties in a single research paper, or discussed the biological properties in two species, which can mistakenly suggest that there were more than 26 papers under analysis. For ease of reference, Table 8 summarizes the research studies on the MTA HP material.

Table 6. Selected physicochemical parameters of the MTA HP.

\begin{tabular}{|c|c|c|c|c|}
\hline Material Characteristics & $\begin{array}{l}\text { Activation } \\
\text { Ultrasonic }\end{array}$ & Time Period & $\begin{array}{c}\text { Value } \\
\text { (Mean } \pm \text { Standard } \\
\text { Deviation) }\end{array}$ & Source \\
\hline \multirow{4}{*}{ Setting time (min) } & no & $120 \pm 10 s$ & $12.20 \pm 1.09$ & \multirow{2}{*}{ Acris De Carvalho et al., 2021 [32] } \\
\hline & yes & $120 \pm 10 s$ & $10.00 \pm 0.70$ & \\
\hline & - & $30 \mathrm{~s}$ intervals & $13.0 \pm 1.0$ & Galarça et al., 2018 [29] \\
\hline & - & - & $85 \pm 2.64$ & Guimarães et al., 2018 [30] \\
\hline \multirow{3}{*}{ Flow $(\mathrm{mm})$} & no & $180 \pm 5 \mathrm{~s}$ & $9.98 \pm 0.18$ & \multirow{2}{*}{ Acris De Carvalho et al., 2021 [32] } \\
\hline & yes & $180 \pm 5 \mathrm{~s}$ & $10.95 \pm 0.14$ & \\
\hline & & $10 \min$ & $18.15 \pm 1.10$ & Galarça et al., 2018 [29] \\
\hline \multirow{2}{*}{ Dimensional change (\%) } & no & 30 days & $1.72 \pm 0.62$ & \multirow{4}{*}{ Acris De Carvalho et al., 2021 [32] } \\
\hline & yes & 30 days & $3.67 \pm 1.97$ & \\
\hline \multirow{4}{*}{ Solubility (\%) } & no & $24 \mathrm{~h}$ & $-3.66 \pm 1.01$ & \\
\hline & yes & $24 \mathrm{~h}$ & $-0.86 \pm 0.89$ & \\
\hline & - & $24 \mathrm{~h}$ & $-2.77 \pm 1.18$ & Galarça et al., 2018 [29] \\
\hline & - & - & $8.18 \pm 1.74$ & Guimarães et al., 2018 [30] \\
\hline \multirow{2}{*}{ Water absorption (\%) } & - & $24 \mathrm{~h}$ & $16.32 \pm 2.92$ & Galarça et al., 2018 [29] \\
\hline & - & - & $14.96 \pm 0.95$ & Guimarães et al., 2018 [30] \\
\hline \multirow{3}{*}{$\mathrm{Ca}_{2}+$ ions release $(\mathrm{mg} / \mathrm{L})$} & no & - & $28.76 \pm 0.93$ & Acris De Carvalho et al., 2021 [32] \\
\hline & yes & - & $35.85 \pm 5.07$ & \\
\hline & - & - & $14.80 \pm 1.58$ & Guimarães et al., 2018 [30] \\
\hline \multirow{2}{*}{ Bond strength (Mpa) } & no & - & $2.54 \pm 1.26$ & \multirow{2}{*}{ Aguiar et al., 2019 [31] } \\
\hline & yes & - & $4.13 \pm 2.43$ & \\
\hline $\begin{array}{l}\text { Film thickness }(\mu \mathrm{m}) 150 \mathrm{~N} \\
\text { was applied }\end{array}$ & - & $10 \mathrm{~min}$ & $194 \pm 89$ & Galarça et al., 2018 [29] \\
\hline Exterior Volume (cm3) & - & - & $0.0877 \pm 0.0045$ & \multirow{4}{*}{ Guimarães et al., 2018 [30] } \\
\hline Volume of Open Pores (cm3) & - & - & $0.0258 \pm 0.0006$ & \\
\hline $\begin{array}{l}\text { Volume of Impervious } \\
\text { Portion }(\mathrm{cm} 3)\end{array}$ & - & - & $0.0619 \pm 0.0044$ & \\
\hline Apparent Porosity (Vop/V \%) & - & - & $29.45 \pm 1.49$ & \\
\hline
\end{tabular}


Table 6. Cont.

\begin{tabular}{|c|c|c|c|c|}
\hline Material Characteristics & $\begin{array}{l}\text { Activation } \\
\text { Ultrasonic }\end{array}$ & Time Period & $\begin{array}{c}\text { Value } \\
\text { (Mean } \pm \text { Standard } \\
\text { Deviation) }\end{array}$ & Source \\
\hline Failure type- Adhesive (\%) & no & - & 56.25 & \multirow{16}{*}{ Aguiar et al., 2019 [31] } \\
\hline Failure type- Adhesive (\%) & yes & - & 25 & \\
\hline Failure type- Cohesive (\%) & no & - & 37.5 & \\
\hline Failure type- Cohesive (\%) & yes & - & 25 & \\
\hline $\begin{array}{l}\text { Failure type-Mixed (adhesive and } \\
\text { cohesive) }(\%)\end{array}$ & yes & - & 6.25 & \\
\hline $\begin{array}{l}\text { Failure type-Mixed (adhesive and } \\
\text { cohesive) }(\%)\end{array}$ & no & - & 50 & \\
\hline $\begin{array}{l}\text { interface of adaptation to the } \\
\text { dentin wall (\% of gaps) }\end{array}$ & no & - & $\begin{array}{l}28.58 \text { (8.01-63.73) } \\
\text { Median (min.-max.) }\end{array}$ & \\
\hline $\begin{array}{l}\text { interface of adaptation to the } \\
\text { dentin wall (\% of gaps) }\end{array}$ & yes & - & $\begin{array}{c}17.87(0.0-43.26) \\
\text { Median (min.-max.) }\end{array}$ & \\
\hline \multirow{8}{*}{ discoloration } & yes & 7 days & $2.77 \pm 1.12$ & \\
\hline & no & 7 days & $1.95 \pm 1.38$ & \\
\hline & yes & 15 days & $2.70 \pm 0.74$ & \\
\hline & no & 15 days & $1.75 \pm 1.46$ & \\
\hline & yes & 30 days & $3.26 \pm 1.47$ & \\
\hline & no & 30 days & $1.44 \pm 1.33$ & \\
\hline & yes & 180 days & $2.60 \pm 0.79$ & \\
\hline & no & 180 days & $1.68 \pm 0.67$ & \\
\hline \multirow{3}{*}{$\begin{array}{l}\text { Evaluation of Microleakage values } \\
\text { of leaked glucose }(\mathrm{mM} / \mathrm{L})\end{array}$} & - & 1 day & $0.083 \pm 0.005$ & \multirow{3}{*}{ Çırakoğlu et al., 2020 [33] } \\
\hline & - & 10 days & $3.644 \pm 6.164$ & \\
\hline & - & 20 days & $5.043 \pm 3.663$ & \\
\hline \multirow{2}{*}{ Radiopacity (mm/Al) } & - & - & $3.04 \pm 0.16$ & Galarça et al., 2018 [29] \\
\hline & - & - & $4.50 \pm 0.46$ & Guimarães et al., 2018 [30] \\
\hline
\end{tabular}


Table 7. Selected biological parameters of the MTA HP.

\begin{tabular}{|c|c|c|c|c|c|c|c|c|}
\hline Biological Parameter & $\begin{array}{l}\text { Additional } \\
\text { Parameter }\end{array}$ & Results & Type of Research & Period Time & Tissue/Cell & Species & Number of Samples & Source \\
\hline \multirow{4}{*}{$\begin{array}{l}\text { Inflammatory } \\
\text { response }\end{array}$} & - & $\begin{array}{l}\text { no activation of THP-1 monocytes } \\
\text { not alter MMP- } 2 \text { and MMP-9 } \\
\text { protein expression }\end{array}$ & In vitro & $48 \mathrm{~h}$ & $\begin{array}{l}\text { monocytes and } \\
\text { macrophages from } \\
\text { THP-1 cells }\end{array}$ & Human & - & Barczak et al., 2021 [12] \\
\hline & - & moderate-few inflamation & \multirow{3}{*}{ In vivo } & 30-90 days & \multirow{3}{*}{$\begin{array}{l}\text { skin and } \\
\text { subcutaneous tissues }\end{array}$} & \multirow{3}{*}{ Wistar rats } & 8 & Benetti et al., 2019 [21] \\
\hline & - & no response & & 30 days & & & 8 & Benetti et al., 2018 [20] \\
\hline & $\begin{array}{l}\text { Hypertension } \\
\text { Normo-tention }\end{array}$ & $\begin{array}{l}\text { increase inflammation } \\
\text { decrease inflammation }\end{array}$ & & 30 days & & & $\begin{array}{c}16 \text { sponta-neously } \\
\text { hyper-tensive (SHR) } 16 \\
\text { normo-tensive (NT) }\end{array}$ & Cosme-Silva et al., 2019 [18] \\
\hline \multirow{7}{*}{ Cytotoxicity } & - & $\begin{array}{l}\text { cell viability similar or higher than the } \\
\text { negative control }\end{array}$ & \multirow{6}{*}{ In vitro } & $24 \mathrm{~h}$ & osteoblastic cells & \multirow{5}{*}{ Human } & 96 plates & Queiroz et al., 2021 [25] \\
\hline & - & $\begin{array}{l}\text { a significant increase in compare to } \\
\text { control group (coulture complete } \\
\text { medium without MTA HP) }\end{array}$ & & 5 days & dental pulp stem cells & & 21 & El Reash et al. 2021 [28] \\
\hline & - & no cytotoxic effect & & $72 \mathrm{~h}$ & dental pulp stem cells & & 10 & Tomás-Catalá et al., 2017 [23] \\
\hline & $\begin{array}{c}\mathrm{pH} 5,2 \\
\text { dilutions } \\
1: 1 \\
1: 2 \\
1: 4\end{array}$ & $\begin{array}{l}\text { depends on the } \mathrm{pH} \text { and degree of } \\
\text { material dilution. }\end{array}$ & & $72 \mathrm{~h}$ & $\begin{array}{l}\text { periodontal ligament } \\
\text { stem cells }\end{array}$ & & 10 & Collado-Gonzlez et al., 2016 [26] \\
\hline & & $\begin{array}{l}\text { depends on the degree of material } \\
\text { dilution. }\end{array}$ & & $72 \mathrm{~h}$ & $\begin{array}{l}\text { bone primary } \\
\text { osteoblasts }\end{array}$ & & 10 & Ferreira et al. 2019 [6] \\
\hline & & $\begin{array}{l}\text { depends on the degree of material } \\
\text { dilution. }\end{array}$ & & $48 \mathrm{~h}$ & Fibroblast-like cells & Mouse & 96 plates & Benetti et al., 2019 [21] \\
\hline & & discrete inflammation & In vivo & 30 days & $\begin{array}{l}\text { skin and subcutaneous } \\
\text { tissues }\end{array}$ & Wistar rats & 10 & Ferreira et al. 2019 [6] \\
\hline \multirow{3}{*}{ Apoptosis/necrosis } & - & $>94 \%$ of viable cells & \multirow{2}{*}{ In vitro } & $72 \mathrm{~h}$ & $\begin{array}{l}\text { bone primary } \\
\text { osteoblasts }\end{array}$ & \multirow{2}{*}{ Human } & 10 & Ferreira et al. 2019 [6] \\
\hline & & $96 \%$ of viable cells & & $72 \mathrm{~h}$ & $\begin{array}{l}\text { periodontal ligament } \\
\text { stem cells }\end{array}$ & & 10 & Collado-Gonzlez et al., 2016 [26] \\
\hline & - & discrete & In vivo & 4 weeks & $\begin{array}{l}\text { Skin and ubcutaneous } \\
\text { tissues }\end{array}$ & Wistar rats & 25 & Elreash et al., 2019 [9] \\
\hline
\end{tabular}


Table 7. Cont.

\begin{tabular}{|c|c|c|c|c|c|c|c|c|}
\hline Biological Parameter & $\begin{array}{l}\text { Additional } \\
\text { Parameter }\end{array}$ & Results & Type of Research & Period Time & Tissue/Cell & Species & Number of Samples & Source \\
\hline \multirow{4}{*}{$\begin{array}{l}\text { Cell attachment } \\
\text { on material }\end{array}$} & $\begin{array}{c}\text { dilutions } \\
1: 1 \\
1: 2 \\
1: 4\end{array}$ & good attachment to the material & \multirow{4}{*}{ In vitro } & $72 \mathrm{~h}$ & $\begin{array}{c}\text { bone primary } \\
\text { osteoblasts }\end{array}$ & \multirow{4}{*}{ Human } & 10 & Ferreira et al. 2019 [6] \\
\hline & - & $\begin{array}{l}\text { Worse attachment than Neo MTA and } \\
\text { Biodentine }\end{array}$ & & $72 \mathrm{~h}$ & dental pulp stem cells & & 10 & Tomás-Catalá et al., 2017 [23] \\
\hline & - & good attachment to the material & & 7 days & dental pulp stem cells & & 21 & El Reash et al. 2021 [28] \\
\hline & $\begin{array}{c}\mathrm{pH} 5,2 \\
\text { dilutions } \\
1: 1 \\
1: 2 \\
1: 4\end{array}$ & $\begin{array}{l}\text { non depends on } \mathrm{pH} \text { and degree of } \\
\text { material dilution. }\end{array}$ & & $72 \mathrm{~h}$ & $\begin{array}{l}\text { periodontal ligament } \\
\text { stem cells }\end{array}$ & & 10 & Collado-Gonzlez et al., 2016 [26] \\
\hline \multirow{4}{*}{ Minera-lization } & & $\begin{array}{l}\text { significant increases in the amounts of } \\
\text { mineralizing nodules formed compare } \\
\text { to control group }\end{array}$ & \multirow{2}{*}{ In vitro } & 7 days & dental pulp stem cells & \multirow{2}{*}{ Human } & 21 & El Reash et al. 2021 [28] \\
\hline & & $\begin{array}{l}\text { more mineralization noodles than } \\
\text { control group }\end{array}$ & & $24 \mathrm{~h}$ & osteoblastic cells & & 96 plates & Queiroz et al., 2021 [25] \\
\hline & $\begin{array}{l}\text { Hypertension } \\
\text { Normo-tension }\end{array}$ & $\begin{array}{l}\text { decrease biomineralization increase } \\
\text { biomineralization }\end{array}$ & \multirow{2}{*}{ In vivo } & 30 days & $\begin{array}{l}\text { skin and subcutaneous } \\
\text { tissues }\end{array}$ & \multirow{2}{*}{ Wistar rats } & $\begin{array}{c}16 \text { spontaneously } \\
\text { hypertensive (SHR) } 16 \\
\text { normotensive (NT) }\end{array}$ & El Reash et al. 2021 [28] \\
\hline & & $100 \%$ biomineralization ability & & 30 days & $\begin{array}{l}\text { skin and subcutaneous } \\
\text { tissues }\end{array}$ & & 8 & Benetti et al. 2018 [20] \\
\hline \multirow[b]{2}{*}{ Antimicrobal effect } & E. faecalis & positive activity & \multirow[b]{2}{*}{ In vitro } & $48 \mathrm{~h}$ & osteoblastic cells & \multirow[b]{2}{*}{ Human } & 96 plates & Queiroz et al., 2021 [25] \\
\hline & $\begin{array}{l}\text { E. faecalis } \\
\text { E. faecum } \\
\text { S. aureus } \\
\text { P. gingivalis } \\
\text { C. albicans } \\
\text { A. israelii } \\
\text { P. anaerobius } \\
\text { S. mutans }\end{array}$ & $\begin{array}{l}\text { positive activity } \\
\text { positive activity } \\
\text { positive activity } \\
\text { positive activity } \\
\text { positive activity } \\
\text { negative activity } \\
\text { negative activity } \\
\text { negative activity }\end{array}$ & & $48 \mathrm{~h}$ & dental pulp stem cells & & 21 & El Reash et al., 2019 [27] \\
\hline
\end{tabular}


Table 8. Summary of MTA HP articles.

\begin{tabular}{|c|c|c|c|c|c|}
\hline $\mathrm{Nr}$ & Source & In Vitro/In Vivo & $\begin{array}{c}\text { Physicochemical } \\
\text { Properties }\end{array}$ & $\begin{array}{c}\text { Biological } \\
\text { Properities }\end{array}$ & Type of Research \\
\hline 1. & Silva et al., 2016 [5] & In vitro & Bond strength & - & Comparative Study \\
\hline 2. & Galarça et al., 2018 [29] & In vitro & $\begin{array}{c}\text { Film thickness, } \\
\text { flow, } \\
\text { setting time, } \\
\text { compressive strength }\end{array}$ & Cell viability & Research Article \\
\hline 3. & Guimarães et al., 2018 [30] & - & $\begin{array}{l}\text { Radiopacity, } \\
\text { calcium } \\
\text { release, } \\
\text { water sorption, } \\
\text { solubility }\end{array}$ & - & Comparative Study \\
\hline 4. & Aguair et al., 2019 [31] & In vitro & $\begin{array}{c}\text { Bond strength, } \\
\text { marginal adaptation, } \\
\text { tooth discoloration }\end{array}$ & - & Comparative Study \\
\hline 5. & Acris De Carvalho et al., 2021 [32] & - & $\begin{array}{c}\text { Setting time, } \\
\text { flow, } \\
\text { dimensional change, } \\
\text { solubility }\end{array}$ & - & Comparative Study \\
\hline 6. & Çırakoğlu et al., 2020 [33] & In vitro & Microleakage & - & Comparative Study \\
\hline 7. & Meraji et al., 2020 [34] & In vitro & $\begin{array}{l}\text { Microhardness, } \\
\text { microstructure }\end{array}$ & - & Comparative Study \\
\hline 8. & Metlerska et al., 2021 [35] & In vitro & Color Changes & - & Comparative Study \\
\hline 9. & Jiménez-Sánchez et al., 2019 [36] & - & $\begin{array}{l}\text { Chemical } \\
\text { composition, } \\
\text { Hydration } \\
\text { performance }\end{array}$ & - & Research Article \\
\hline 10. & Jiménez-Sánchez et al., 2020 [37] & In vitro & $\begin{array}{l}\text { Hydration } \\
\text { performance }\end{array}$ & Bioactive response & Comparative Study \\
\hline 11. & Jiménez-Sánchez et al., 2019 [38] & In vitro & $\begin{array}{l}\text { Microstructural } \\
\text { features }\end{array}$ & Bioactive response & Research Article \\
\hline 12. & Jiménez-Sánchez et al., 2019 [39] & In vitro & - & Mineralization & Research Article \\
\hline 13. & Barczak et al., 2021 [12] & In vitro & - & $\begin{array}{l}\text { Inflammatory } \\
\text { response }\end{array}$ & Research Article \\
\hline 14. & Tomás-Catalá et al., 2017 [23] & In vitro & $\begin{array}{l}\text { Chemical } \\
\text { composition }\end{array}$ & $\begin{array}{l}\text { Cell viability, } \\
\text { cell migration, } \\
\text { cell morphology, } \\
\text { cell attachment }\end{array}$ & Comparative Study \\
\hline 15. & Tomás-Catalá et al., 2018 [24] & In vitro & $\begin{array}{l}\text { Chemical } \\
\text { composition }\end{array}$ & $\begin{array}{l}\text { Cell viability, } \\
\text { cell migration, } \\
\text { cell morphology, } \\
\text { cell attachment }\end{array}$ & Comparative Study \\
\hline 16. & Queiroz et al., 2021 [25] & In vitro & $\begin{array}{l}\text { Setting time, } \\
\text { radiopacity, } \\
\text { pH, } \\
\text { solubility }\end{array}$ & $\begin{array}{c}\text { Cytotoxicity, } \\
\text { cell bioactivity, } \\
\text { alkaline phosphatase } \\
\text { activity, } \\
\text { Alzarin red staining } \\
\text { (ARS), } \\
\text { real time PCR } \\
\text { (qPCR), } \\
\text { antibacterial activity }\end{array}$ & Comparative Study \\
\hline 17. & Collado-Gonzlez et al., 2016 [26] & In vitro & $\begin{array}{l}\text { Chemical } \\
\text { composition }\end{array}$ & $\begin{array}{c}\text { Cell viability, } \\
\text { aapoptosis, } \\
\text { cell attachment }\end{array}$ & Comparative Study \\
\hline 18. & El Reash et al., 2019 [27] & In vitro & $\mathrm{pH}$ & Antimicrobial effect & Comparative Study \\
\hline 19. & El Reash et al., 2021 [28] & In vitro & - & $\begin{array}{l}\text { Cytotoxicity, } \\
\text { Cell attachment, } \\
\text { mineralization }\end{array}$ & Comparative Study \\
\hline
\end{tabular}


Table 8. Cont.

\begin{tabular}{|c|c|c|c|c|c|}
\hline $\mathrm{Nr}$ & Source & In Vitro/In Vivo & $\begin{array}{l}\text { Physicochemical } \\
\text { Properties }\end{array}$ & $\begin{array}{c}\text { Biological } \\
\text { Properities }\end{array}$ & Type of Research \\
\hline 20. & Benetti et al., 2019 [21] & In vitro/In vivo & - & $\begin{array}{c}\text { Cytotoxicity, } \\
\text { biocompatibility, } \\
\text { biomineralization }\end{array}$ & Comparative Study \\
\hline 21. & Benetti et al., 2018 [20] & In vivo & - & $\begin{array}{l}\text { Biocompatibility, } \\
\text { biomineralization }\end{array}$ & Comparative Study \\
\hline 22. & Ferreira et al., 2019 [6] & In vivo & $\begin{array}{l}\text { Setting time, } \\
\text { flow, } \\
\text { radiopacity, } \\
\text { solubility }\end{array}$ & $\begin{array}{l}\text { Cytotoxicity, } \\
\text { Apoptosis, } \\
\text { cell adhesion }\end{array}$ & Comparative Study \\
\hline 23. & El Reash et al., 2019 [9] & In vivo & - & $\begin{array}{l}\text { Inflammatory } \\
\text { response }\end{array}$ & Comparative Study \\
\hline 24. & Cosme-Silva et al., 2019 [18] & In vivo & - & $\begin{array}{l}\text { Biocompatibility, } \\
\text { biomineralization }\end{array}$ & Comparative Study \\
\hline 25. & Delfino et al., 2021 [19] & In vivo & - & $\begin{array}{c}\text { Bioactivity, } \\
\text { biocompatibility }\end{array}$ & Comparative Study \\
\hline 26. & Macedo et al., 2020 [22] & In vivo & - & Biomineralization & Research Article \\
\hline
\end{tabular}

\section{Discussion}

MTA-based materials are classified as bioceramic materials. Generally, bioceramic materials are biocompatible ceramic compounds originating both in situ as well as in vivo from various chemical processes. They show superior biocompatibility due to biological characteristics resembling those of hydroxyapatite. During hydration, bioceramics generate numerous compounds, e.g., hydroxyapatite, which show the ability to induce regenerative reaction in the human organism. At contact with the bone, mineral hydroxyapatite shows osteoconductive properties which results in the formation of a new bone in the interphase. Additionally, bioceramic materials exhibit inherent osteoinductive ability due to the reported ability to absorb osteoinductive substances near the bone healing site $[1,2]$. Bioceramic materials are biocompatible and have antibacterial properties. The latter is due to in situ precipitation of the material, following the setting time, which results in bacteria sequestration. The materials take the form of porous preparation with nanocrystals of 1-3 nm in diameter, thus preventing bacteria adhesion. At times, fluoride ions are the constituents of apatite crystal, and the resulting nanomaterial shows antibacterial properties. Furthermore, bioceramics can be used jointly with synthetic hydroxyapatite [1,4]. The MTA HP cement was developed to overcome the disadvantages of previous MTA materials. In particular, the materials used for retrofilling the canals require greater plasticity, shorter setting time and easy insertion into the root [12]. The discussion describes the selection of the most important physicochemical and biological parameters of the MTA HP as a bioceramic material. Whenever possible, the properties of the HP MTA material were compared to other MTA materials. The most significant physicochemical parameters with respect to results obtained by different authors are presented in Tables 6-8.

\subsection{Physicochemical Properties}

\subsubsection{Setting Time}

The setting time of endodontic cement should be less than $10 \%$ of the time specified by the manufacturer. The classic calcium-silicate materials dissolve in tissues after some time following the application, which is an undesirable feature of these materials. However, unlike calcium hydroxide, MTA HP shows very low solubility [13]. According to Galarça et al. in 2018 [29], the MTA repair HP cement had a longer setting time than MTA Angelus. In the MTA HP material, calcium tungstate was used as a radiation sedative instead of bismuth oxide [29]. The use of calcium tungsten is beneficial because it has a stimulating effect on the physicochemical, antibacterial and biological properties of MTA cement [40]. Additionally, in the case of MTA HP, it is assumed that the plasticizer included 
in the liquid formulation of this cement favored the reduction of the setting time [29]. In turn, Jiménez-Sánchez (2019) stated that the short setting time measured for MTA HP is correlated with the precursor powder high surface area, the absence of compositional sulphate phases, and high $\mathrm{Al}$ content. Calcium sulphates affect the rate of chemical bonding. The less sulphate, the shorter the setting time of the MTA HP material [36]. This is contrary to the findings of Guimarães et al., 2018, which showed that MTA HP showed similar end-setting time values as MTA Angelus, but that the presence of a plasticizer could increase solubility and porosity [30]. However, Acris De Carvalho et al., 2021, indicated that when MTA HP was activated by ultrasound, the setting time was reduced when compared to the control group of MTA HP without ultrasound activation. The authors provide the following explanation: when ultrasonic activation is used, there is an air vortex and changes in hydrostatic pressure, which favors the formation of cavitation, causing an increase in the temperature of the material. This increases the hydration and reaction kinetics $[32,41]$. The presence of water in the cement leads to the transformation of calcium oxide into calcium hydroxide and, additionally, shortens the setting time [42].

\subsubsection{Flow and Dimensional Change}

Endodontic cements cannot have a diameter smaller than $20 \mathrm{~mm}$ in the flow test [43]. According to the study by Acris De Carvalho et al. (2021) MTA HP met the standard only following activation with ultrasound [32]. Chemical transformations occurring as a result of the material's contact with water in the process of cement hydration and an increase in temperature may affect the decrease in cement particle diameter and the reduction of the viscosity of the cements, favoring the flow [44]. In turn, as pointed out by Bodanezi et al. in 2008, the changes in the particle size contribute to a decrease in sealing ability of the cement and may favor the development of infection, thus leading to treatment failure [45].

\subsubsection{Solubility and Water Sorption}

As for solubility, testing observed that it should not exceed $3 \%$ of the material's mass [43]. In the case of MTA HP, following the preparation of the material according to the manufacturer's instructions, there is an increase in mass due to water absorption. The pores form on the surface as well as inside the material. These empty spaces weaken the structure of the cement and are susceptible to the activity of the solvent which allows water absorption [46]. The conventional material of the MTA was compared with that of the MTA HP. It was found that conventional MTA showed the lowest values for solubility, open pore volume, apparent porosity and water sorption in comparison to MTA HP [30]. This difference between the conventional MTA and MTA HP, both of which have similar compositions, could be due to the plasticizer contained in the mixing liquid of MTA HP [30] Hydration contributes to increasing the mass of cement, loosening its structure and creating voids $[29,47]$. Ultrasonic activation reduces the occurrence of this unfavorable phenomenon [32].

\subsubsection{Film Thickness, Bond and Compressive Strength}

With respect to film thickness, Galarça et al. (2018) stated that MTA HP had a significantly lower film thickness (but it still did not meet the ISO 6876 standard) than MTA Angelus. This property may be explained by observations that are in agreement regarding a finer particle size. Moreover, it was also found that MTA HP compared to conventional MTA and Biodentine shows lower bond strength and marginal adaptation that can be enhanced by ultrasound [31]. The particle size of the new cement was slightly smaller than that of MTA Angelus [29]. However, a study by Silva et al. in 2016 showed a new methodological aspect. Pores were made in the dentin fragments and care was taken to ensure that the MTA HP material and time points were further compared using the same dentin sample $[5,48]$. The authors concluded that HP MTA showed better bond strength and resistance to thrust compared to white MTA but worse than Biodentine. Replacing bismuth oxide with calcium tungstate as a radiopacificator in MTA HP could explain the better 
performance of this cement compared to white MTA [5]. Calcium tungstate contributes to higher calcium release, promoting higher biomineralization [49]. Regarding compressive strength, when compared with the conventional MTA, lower values were recorded for MTA HP following $24 \mathrm{~h}$, and the values showed an increase with time and were higher than that of the conventional MTA following 28 days. The improvement of compressive strength in time may reduce susceptibility to fracturing and cracking of the cement. This is a desired feature as it makes the material more durable to occlusal stress [29].

\subsubsection{Marginal Adaptation and Microleakage}

It was found that the acoustic force induced by ultrasounds has a positive effect on the adaptation of the material to the walls of root canals. Therefore, better adaptation of the MTA HP material increases the adhesion [30].

According to the results by Aguiar et al., 2019, MTA HP maintains the color stability and does not result in tissue discolouration owing to lack of bismuth oxide. This feature is an undisputed advantage over the conventional MTA containing bismuth oxide as a radiopacifier [31]. Metlerska et al. (2021) analyzed the effects of $10 \%$ and $40 \%$ citric acid (CA) on the color of calcium silicate-based cements (CSCs) in comparison to the effects of common root canal irrigants [35]. It was found that MTA repair HP was least sensitive to $\mathrm{NaOCl}$ solution of all the tested CSCs materials. It also exhibited the slightest spectrophotometric color changes upon immersion in CHX and EDTA. Only in contact with CA solution did its surface structure disintegrate. Irrigation solutions do not cause darkening of MTA HP, which could be visible to the naked eye [35].

\subsubsection{Main Compositional Phases and $\mathrm{pH}$}

The main components of MTA HP are: $\mathrm{CaWO}_{4}, \mathrm{Ca}_{3} \mathrm{SiO}_{5}$ and $\mathrm{Ca}_{2} \mathrm{SiO}_{4}$ as the main phases of the composition. The content of calcium aluminate improves the biological response of MTA HP [27,36]. In several scientific studies, the chemical composition of HP MTA was determined using energy dispersion X-rays $[14,15,17,36]$. Jiménez-Sánchez et al. (2020) found that the structure of the MTA HP tricalcium silicate particles ensures a very close contact between the calcium silicate and calcium aluminate and thus favors the hydration reaction. In this way, hydrated calcium aluminate silicate is formed which is responsible for the biocompatibility and speed of the reaction [38]. El Reash et al. (2019) found that MTA HP was less alkaline $(\mathrm{pH}=11.5)$ compared to iRoot BP Plus ( $\mathrm{pH} 12.1)$ [27].

\subsubsection{The Release of Calcium Ions}

An important aspect of endodontic treatment is the proper formation of mineralized tissue. Biomineralization is the synthesis of a mineral by a living organism. In turn, bioactivity is the deposition of calcium phosphate deposits on the surface of materials in the buffer. The buffer contains ions close to human plasma [50-52]. The most important thing in the pulp capping is the release of calcium ions. Calcium influences the differentiation of pulp cells and thus the mineralization of hard tissue $[52,53]$. MTA HP releases calcium most intensively in the first month after application, compared to conventional MTA, which showed the highest release of calcium after 1 week [30]. The release of calcium ions takes place by dissociation of calcium hydroxide, the by-product of which is the hydration of $\mathrm{Ca}_{2}+$ and $\mathrm{OH}-$ ions, tricalcium and dicalcium [54].

The growth of calcium phosphate apatite crystals is the environment for the differentiation and colonization of stem cells and osteoblasts [5]. Scanning electron microscopy analysis provided qualitative and semi-quantitative measurements of calcium and atomic phosphorus. The high intensity of the $\mathrm{Ca}$ and $\mathrm{P}$ peaks detected in the analysis indicated the precipitation of amorphous sediments corresponding to calcium phosphate [55]. The Ca:P atomic ratio one month after application was lower for MTA HP material compared to conventional MTA. This result may be correlated with the presence of a plasticizer [30]. On the other hand, the MTA HP material is more effective regarding the bioactive reaction rate compared to the Pro and Neo materials [30]. This is in line with the studies 
by Jiménez-Sánchez et al., who conducted an in vitro bioactivity assessment of MTA HP material. The authors believe that MTA HP can be used as an endodontic repair cement due to its high biocompatibility [39].

\subsection{Biological Properties}

\subsubsection{Inflammatory Response}

Out of the biological parameters, the inflammatory response of the tissues in contact with MTA HP is discussed particularly often $[6,9,12,18,20,21]$. From the clinical perspective, the most desirable situation is that of a lack of such a response, which would indicate an ideal biocompatibility of the material covering the tissue. However, the research papers under analysis show that MTA HP causes a local inflammatory reaction in contact with living tissue. The in vivo studies conducted on rats showed that MTA HP was implanted in the dorsal region for some time. Next, having euthanized the animals, the tissue with an implanted MTA HP was assessed in terms of inflammatory reaction $[6,9,18,20,21]$. The most severe inflammatory reaction was found in the first few days following the contact of the tissue with MTA HP and showed a decrease in time. After 30 days, the inflammatory response was found to be slight $[6,9,20,21]$. However, none of the research articles under study provides an assessment of the final resolution of the inflammatory response. The longest experiment (90 days) was conducted by Benetti et al. in 2018. Yet, even after such a long time, the authors still recorded the presence of single inflammatory cells [20]. An interesting observation was made by Cosme-Silva et al., 2019, who observed an increase in the tissue inflammatory infiltrate at contact with MTA HP in rats with spontaneous hypertension as compared to rats with normal blood pressure [18]. The authors note that the host's response to substances released by the materials is a major factor influencing the progression and type of inflammatory infiltrate. Immune cells release cytokines and growth factors that play a key role in the inflammatory process. However, the inflammatory response of tissues in contact with cement may be exacerbated in hypertension. The reason for this is an increase in lymphocyte infiltration and multiplication, and an increased concentration of pro-inflammatory cytokines: interleukin-1 beta (IL-1 $\beta$ ), interleukin-6 (IL6) and tumor necrosis factor alpha (TNF- $\alpha$ ) [18]. Barczak et al., 2021 investigated the MTA HP formula with respect to its effect on the inflammation process involving the tooth and periodontal tissues. The experiments were conducted in vitro on human monocytes of THP1 cell line and macrophages obtained from THP- 1 cells. It was found that MTA HP showed no activation of THP- 1 monocytes and did not alter MMP-2 and MMP-9 protein expression in the cultured monocytes/macrophages. The crucial regulatory role of MMPs in terms of cell differentiation and migration processes, growth factors, angiogenesis, as well as development of inflammation has been confirmed [12]. Additionally, the inflammatory reaction caused by MTA HP was compared to that of other bioceramic materials. In the study by Benetti et al., 2019, it was found that in the group of rats with MTA HP and white MTA-Angelus implanted into the subcutaneous tissue, most of the samples had a mild inflammatory infiltrate [21]. However, in the group of rats with an implanted Bio-C Repair, there was only a mild to absent inflammatory reaction. Delfino et al., 2021 [19], assessed the inflammatory response elicited by Bio-C Pulpo, MTA HP and white MTA [19]. The authors concluded that the materials did not promote an inflammatory response and that the materials were biocompatible [19]. However, it must be observed that none of the aforementioned research papers on inflammatory response to MTA HP provides the time after which the inflammatory reaction recedes following the contact with MTA HP.

\subsubsection{Cytotoxicity}

Another parameter discussed in the literature on the subject and analysed in the present paper is the cytotoxicity of MTA HP. This parameter is determined in most publication with MTT (3-[4,5-dimethylthiazol-2-yl]-2,5 diphenyl tetrazolium bromide) assay [56]. Ferreira et al. (2019) and Benetti et al. (2019) found that in vitro, cell viability depends on the degree of material dilution. The less the material is diluted, the less viable are the 
cells [6,21]. It is worth mentioning that the results are similar with respect to mice cells (fibroblast-like cells) as well as human cells (NHOst human bone primary osteoblasts) $[6,21]$. El Reash et al., 2021, investigated the effects of MTA HP on the proliferation of human Dental Pulp Stem Cells (hDPSCs) and found a significant increase in hDPSCs cellular proliferation as compared to control group (culture complete medium without MTA HP) [29]. This may be due to the leaching of calcium ions [28]. The released calcium is conducive to apatite formation: the tricalcium silicate- based cements, when in contact with interstitial fluid, released calcium, silicate hydrate and calcium hydroxide [57]. Calcium hydroxide releases calcium ions necessary for cells adhesion, migration, differentiation and proliferation of cells. Moreover, calcium plays a key role in fibroblast adhesion [58,59]. It was proved that more Ca ions are released from MTA HP at $\mathrm{pH} 5.2$ than at $\mathrm{pH} 7.4$ [26]. The explanation of the relationship is not clear due to insufficient number of analyses of MTA $\mathrm{HP}$ in contact with an acidic environment. Additionally, Tomás-Catalá at al. studied evaluate the in vitro cytotoxicity of MTA HP, Neo MTA Plus, and Biodentine, on human dental pulp stem cells (hDPSCs) [24]. The cytotoxicity of MTA HP, Neo MTA Plus and Biodentine materials was compared. It was found that all tested materials showed a normal level of cytocompatibility with hDPSC cells and good cell migration rates, although the best parameters were obtained for Biodentine [24].

\subsubsection{Apoptosis/Necrosis}

Several authors are in line with respect to low ability of MTA HP to generate apoptosis of cells in contact with the material. The estimated cell viability following the contact with MTA HP is more than $90 \%[6,9,26]$.

\subsubsection{Cellular Adhesion}

The MTT test is an adequate method of quantitative assessment of cell viability/proliferation [26]. However, it only provides an investigation of a single aspect of biocompatibility and is not to be used alone to determine if the material is cytocompatible or not [60]. Therefore, apart from MTT test, the ability of the material to provide an adequate surface for cellular adhesion is analyzed with the scanning electron micrographs (SEM), the extracellular calcium deposition (bioactivity) is quantitatively assessed with Alizarin Red staining to fully understand the properties of the materials. Research shows that after 3 days of cultivation, hPDLSCs attached to the MTA HP material when exposed to various environments. No differences were observed between the material and the acidic or neutral environment [26]. Additionally, after 72 h, Ferreira et al., 2019 observed a large number of cells covering the disc surface coated with MTA HP and cultured cells [6]. However, as has been demonstrated by El Reash et al., 2021, after 7 days, the cells (human hDPSCs) exhibited characteristics of fibroblastic morphology, reflecting good attachment to the MTA HP [28]. On the other hand, however, it must be observed that bioceramic materials show different adhesion to tissues, as has been demonstrated by [23]. The authors compared the properties of MTA HP, Neo MTA and Biodentine on hDPSCs cells obtained from impacted third molars. They found that MTA HP causes a significantly lower cell adhesion to the material when compared to Neo MTA and Biodentine [23]. The authors attribute this to differences in chemical composition of the elements between the analyzed materials. The presence of $\mathrm{Sr}$ in eluates is 10-times greater in MTA HP and 100-times greater in Neo MTA Plus as compared with Biodentine [23]. The presence of $\mathrm{Sr}, \mathrm{Al}$ and $\mathrm{S}$ affects the cytotoxicity (in Neo MTA Plus and MTA HP), whereas their low amounts or lack (in the case of Biodentine) is connected with a markedly increased biocompatibility of the material [23].

\subsubsection{Mineralization}

In a test with the use of Alizarin Red staining it was found that after $24 \mathrm{~h}$ in human osteoblastic cells there were more mineralization noodles than in the control group (culture complete medium without MTA HP) [25]. A significant increase in the amounts of mineralizing nodules formed, when compared to control group (culture complete medium without 
MTA HP), was also observed following 7 days in dental pulp stem cells (hDPSCs) [28]. In vitro tests conducted on rat cells demonstrated that MTA HP showed a superior mineralization ability of close to $100 \%[25,27]$. It must be noted that some factors, such as arterial hypertension, contributed to a decrease in mineralization ability of the tissues covered with MTA HP [18]. Macedo et al., 2020 investigated the response of the laser photobiomodulation after filling a bone defect with MTA HP in a rat. They found that the irradiated group presented a clear narrowing of the medullary spaces, suggesting greater bone compaction. The authors conclude that laser photobiomodulation, when associated with the use of material MTA HP, has been good for the bone repair process [22].

\subsubsection{Antibacterial Effect}

In terms of the antibacterial effect, MTA HP was assessed against other materials such as ACTIVA or iRoot BP [9]. With respect to facultatively aerobic Gram + ve (S. aureus, S. mutans, E. faecalis and E. faecium), MTA HP showed a marked inhibitory effect on S. aureus, S. mutans and E. faecium, however the effect was found to be even stronger regarding iRoot BP. Similarly, IRoot BP showed the superior antibacterial effect against $E$. faecalis. In terms of P. anaerobius, $A$. israelii and P. gingivalis, again iRoot BP as well as MTA HP showed an inhibitory activity greater than that of ACTIVIA. However, the inhibitory effect of MTA HP on anaerobes was found to be lower than that of iRoot BP. Additionally, it must be emphasized that MTA HP shows no antibacterial properties against $A$. israelii and $P$. gingivalis. Finally, MTA HP demonstrated a superior inhibitory effect on C. albicans when compared to iRoot BP, whereas ACTIVIA showed no antifungal activity [9]. According to the results of other studies, it was found that MTA HP exhibits antimicrobial activity mainly due to its high $\mathrm{pH}$. However, it turns out that the MTA HP material is not effective against anaerobic bacteria [27].

Bioactive calcium-silicate cements are being increasingly used with a widening application options in endodontics and regenerative endodontics. There are a number of research studies describing in detail the physical and chemical properties of MTA HP. It transpires that an improvement of some chemical and biological properties has a negative effect on the physical parameters of the said material. A small number of studies provides the analysis of MTA HP in vivo in human teeth in clinical settings. Most research studies published to date is based on in vitro studies on laboratory animals (mice and rats).

\section{Conclusions}

The analysis of the conducted studies allows for the gaining of greater understanding by doctors prior to using the preparation and, consequently, the obtainment of improved treatment outcomes in clinical settings. However, more long-term in vivo studies with a larger sample size and proper clinical settings are required to extend the knowledge and draw a definitive conclusion on MTA HP.

Funding: This research received no external funding.

Institutional Review Board Statement: Not applicable.

Informed Consent Statement: Not applicable.

Data Availability Statement: Data sharing is not applicable to this article.

Conflicts of Interest: The authors declare no conflict of interest.

\section{References}

1. Jitaru, S.; Hodisan, I.; Timis, L.; Lucian, A.; Bud, M. The use of bioceramics in endodontics-literature review. Med. Pharm. Rep. 2016, 89, 470-473. [CrossRef]

2. Emara, R.; Elhennawy, K.; Schwendicke, F. Effects of calcium silicate cements on dental pulp cells: A systematic review. J. Dent. 2018, 77, 18-36. [CrossRef] [PubMed]

3. Giraud, T.; Jeanneau, C.; Bergmann, M.; Laurent, P.; About, I. Tricalcium Silicate Capping Materials Modulate Pulp Healing and Inflammatory Activity In Vitro. J. Endod. 2018, 44, 1686-1691. [CrossRef] 
4. Sanz, J.L.; Rodríguez-Lozano, F.J.; Llena, C.; Sauro, S.; Forner, L. Bioactivity of Bioceramic Materials Used in the Dentin-Pulp Complex Therapy: A Systematic Review. Materials 2019, 12, 1015. [CrossRef] [PubMed]

5. Silva, E.; Carvalho, N.K.; Zanon, M.; Senna, P.M.; De-Deus, G.; Zuolo, M.L.; Zaia, A.A. Push-out bond strength of MTA HP, a new high-plasticity calcium silicate-based cement. Braz. Oral Res. 2016, 30. [CrossRef] [PubMed]

6. $\quad$ Ferreira, C.M.A.; Sassone, L.M.; Gonçalves, A.S.; De Carvalho, J.J.; Tomás-Catalá, C.J.; García-Bernal, D.; Oñate-Sánchez, R.E.; Rodríguez-Lozano, F.J.; Silva, E. Physicochemical, cytotoxicity and in vivo biocompatibility of a high-plasticity calcium-silicate based material. Sci. Rep. 2019, 9, 3933. [CrossRef]

7. Macwan, C.S.; Deshpande, A. Mineral trioxide aggregate (MTA) in dentistry: A review of literature. J. Oral Res. Rev. 2014,6 , 71. [CrossRef]

8. Kaur, M.; Singh, H.; Dhillon, J.S.; Batra, M.; Saini, M. MTA versus Biodentine: Review of Literature with a Comparative Analysis. J. Clin. Diagn. Res. 2017, 11, ZG01-ZG05. [CrossRef] [PubMed]

9. ElReash, A.A.; Hamama, H.; Abdo, W.; Wu, Q.; El-Din, A.Z.; Xiaoli, X. Biocompatibility of new bioactive resin composite versus calcium silicate cements: An animal study. BMC Oral Health 2019, 19, 1-10. [CrossRef]

10. Kaup, M.; Schäfer, E.; Dammaschke, T. An in vitro study of different material properties of Biodentine compared to ProRoot MTA. Head Face Med. 2015, 11, 1-8. [CrossRef]

11. Kunert, M.; Lukomska-Szymanska, M. Bio-Inductive Materials in Direct and Indirect Pulp Capping-A Review Article. Materials 2020, 13, 1204. [CrossRef]

12. Barczak, K.; Palczewska-Komsa, M.; Lipski, M.; Chlubek, D.; Buczkowska-Radlińska, J.; Baranowska-Bosiacka, I. The Influence of New Silicate Cement Mineral Trioxide Aggregate (MTA Repair HP) on Metalloproteinase MMP-2 and MMP-9 Expression in Cultured THP-1 Macrophages. Int. J. Mol. Sci. 2020, 22, 295. [CrossRef]

13. Tawil, P.Z.; Duggan, D.J.; Galicia, J. Mineral trioxide aggregate (MTA): Its history, composition, and clinical applications. Compend. Contin. Educ. Dent. 2015, 36, 247-264.

14. Casadei, B.D.A.; Lara-Mendes, S.T.O.; Barbosa, C.D.F.M.; Araújo, C.V.; De Freitas, C.A.; Machado, V.C.; Santa-Rosa, C.C. Access to original canal trajectory after deviation and perforation with guided endodontic assistance. Aust. Endod. J. 2020, 46, 101-106. [CrossRef]

15. Page, M.J.; McKenzie, J.E.; Bossuyt, P.M.; Boutron, I.; Hoffmann, T.C.; Mulrow, C.D.; Shamseer, L.; Tetzlaff, J.M.; Akl, E.A.; Brennan, S.E.; et al. The PRISMA 2020 statement: An updated guideline for reporting systematic reviews. Syst. Rev. $2021,10,89$. [CrossRef] [PubMed]

16. Slim, K.; Nini, E.; Forestier, D.; Kwiatkowski, F.; Panis, Y.; Chipponi, J. Methodological index for non-randomized studies (MINORS ): Development and validation of a new instrument. ANZ J. Surg. 2003, 73, 712-716. [CrossRef] [PubMed]

17. Elkhadem, A.; Mickan, S.; Richards, D. Adverse events of surgical extrusion in treatment for crown-root and cervical root fractures: A systematic review of case series/reports. Dent. Traumatol. 2013, 30, 1-14. [CrossRef]

18. Cosme-Silva, L.; Dal-Fabbro, R.; Gonçalves, L.D.O.; Prado, A.S.D.; Plazza, F.A.; Viola, N.V.; Cintra, L.T.A.; Filho, J.E.G. Hypertension affects the biocompatibility and biomineralization of MTA, High-plasticity MTA, and Biodentine®. Braz. Oral Res. 2019, 33, e060. [CrossRef] [PubMed]

19. Delfino, M.; Jampani, J.; Lopes, C.; Guerreiro-Tanomaru, J.; Tanomaru-Filho, M.; Sasso-Cerri, E.; Cerri, P. Comparison of Bio-C Pulpo and MTA Repair HP with White MTA: Effect on liver parameters and evaluation of biocompatibility and bioactivity in rats. Int. Endod. J. 2021. [CrossRef] [PubMed]

20. Benetti, F.; Gomes-Filho, J.E.; Lopes, J.M.A.; Barbosa, J.G.; Jacinto, R.C.; Cintra, L.T.A. In vivo biocompatibility and biomineralization of calcium silicate cements. Eur. J. Oral Sci. 2018, 126, 326-333. [CrossRef] [PubMed]

21. Benetti, F.; Queiroz, ÍO.D.A.; Cosme-Silva, L.; Conti, L.C.; De Oliveira, S.H.P.; Cintra, L.T.A. Cytotoxicity, Biocompatibility and Biomineralization of a New Ready-for-Use Bioceramic Repair Material. Braz. Dent. J. 2019, 30, 325-332. [CrossRef] [PubMed]

22. Macedo, A.A.P.; Santos, T.D.; Cunha, J.L.S.; Matos, F.D.S.; Júnior, R.L.C.D.A.; Ribeiro, M.A.G. Effect of laser photobiomodulation associated with a bioceramic cement on the repair of bone tissue in the femur of rats. J. Photochem. Photobiol. B 2020, $205,111813$. [CrossRef] [PubMed]

23. Tomás-Catalá, C.J.; Collado-González, M.D.M.; García-Bernal, D.; Oñate-Sánchez, R.E.; Forner, L.; Llena, C.; Lozano, A.; CasteloBaz, P.; Moraleda, J.M.; Rodríguez-Lozano, F.J. Comparative analysis of the biological effects of the endodontic bioactive cements MTA-Angelus, MTA Repair HP and NeoMTA Plus on human dental pulp stem cells. Int. Endod. J. 2017, 50, e63-e72. [CrossRef] [PubMed]

24. Tomás-Catalá, C.J.; Collado-González, M.; García-Bernal, D.; Oñate-Sánchez, R.E.; Forner, L.; Llena, C.; Lozano, A.; Moraleda, J.M.; Rodríguez-Lozano, F.J. Biocompatibility of New Pulp-capping Materials NeoMTA Plus, MTA Repair HP, and Biodentine on Human Dental Pulp Stem Cells. J. Endod. 2018, 44, 126-132. [CrossRef] [PubMed]

25. Queiroz, M.B.; Torres, F.F.E.; Rodrigues, E.M.; Viola, K.S.; Bosso-Martelo, R.; Chavez-Andrade, G.M.; Guerreiro-Tanomaru, J.M.; Tanomaru-Filho, M. Physicochemical, biological, and antibacterial evaluation of tricalcium silicate-based reparative cements with different radiopacifiers. Dent. Mater. 2021, 37, 311-320. [CrossRef]

26. Collado-González, M.D.M.; Garcia, S.L.; García-Bernal, D.; Oñate-Sánchez, R.E.; Tomás-Catalá, C.J.; Moraleda, J.M.; Lozano, A.; Forner, L.; Rodríguez-Lozano, F.J. Biological effects of acid-eroded MTA Repair HP and ProRoot MTA on human periodontal ligament stem cells. Clin. Oral Investig. 2019, 23, 3915-3924. [CrossRef] [PubMed] 
27. ElReash, A.A.; Hamama, H.; Eldars, W.; Lingwei, G.; El-Din, A.Z.; Xiaoli, X. Antimicrobial activity and pH measurement of calcium silicate cements versus new bioactive resin composite restorative material. BMC Oral Health 2019, 19, 1-10. [CrossRef]

28. ElReash, A.A.; Hamama, H.; Grawish, M.; Saeed, M.; El-Din, A.M.Z.; Shahin, M.A.; Zhenhuan, W.; Xiaoli, X. A laboratory study to test the responses of human dental pulp stem cells to extracts from three dental pulp capping biomaterials. Int. Endod. J. 2021. [CrossRef]

29. Galarça, A.D.; Rosa, W.L.D.O.D.; Da Silva, T.M.; Lima, G.D.S.; Carreño, N.L.V.; Pereira, T.M.; Guedes, O.A.; Borges, A.H.; da Silva, A.F.; Piva, E. Physical and Biological Properties of a High-Plasticity Tricalcium Silicate Cement. BioMed Res. Int. 2018, 2018, 8063262. [CrossRef]

30. Guimarães, B.M.; Prati, C.; Duarte, M.A.H.; Bramante, C.M.; Gandolfi, M.G. Physicochemical properties of calcium silicate-based formulations MTA Repair HP and MTA Vitalcem. J. Appl. Oral Sci. 2018, 26, e2017115. [CrossRef]

31. Aguiar, B.A.; Frota, L.M.A.; Taguatinga, D.T.; Vivan, R.; Camilleri, J.; Duarte, M.A.H.; De Vasconcelos, B.C. Influence of ultrasonic agitation on bond strength, marginal adaptation, and tooth discoloration provided by three coronary barrier endodontic materials. Clin. Oral Investig. 2019, 23, 4113-4122. [CrossRef]

32. De Carvalho, F.M.A.; Silva-Sousa, Y.T.C.; Miranda, C.E.S.; Calderon, P.H.M.; Barbosa, A.F.S.; De Macedo, L.M.D.; Rached-Junior, F.J.A. Influence of Ultrasonic Activation on the Physicochemical Properties of Calcium Silicate-Based Cements. Int. J. Dent. 2021, 2021, 6697988. [CrossRef]

33. Çırakoğlu, S.; Baddal, B.; Islam, A. The Effectiveness of Laser-Activated Irrigation on the Apical Microleakage Qualities of MTA Repair HP and NeoMTA Plus in Simulated Immature Teeth: A Comparative Study. Materials 2020, 13, 3287. [CrossRef] [PubMed]

34. Meraji, N.; Ahmadi, E. The effect of bleaching agents on the microstructure and surface microhardness of three calcium silicatebased barrier materials. Iran. Endod. J. 2020, 15, 23-30. [CrossRef]

35. Metlerska, J.; Dammaschke, T.; Lipski, M.; Fagogeni, I.; Machoy-Mokrzyńska, A.; Nowicka, A. Effect of Citric Acid on Color Changes of Calcium Silicate-Based Cements an In Vitro Study. Appl. Sci. 2021, 11, 2339. [CrossRef]

36. Jimenez-Sanchez, M.; Segura-Egea, J.; Diaz-Cuenca, A. Physicochemical parameters-hydration performance relationship of the new endodontic cement MTA Repair HP. J. Clin. Exp. Dent. 2019, 11, e739-e744. [CrossRef]

37. Jiménez-Sánchez, M.D.C.; Segura-Egea, J.J.; Díaz-Cuenca, A. Higher hydration performance and bioactive response of the new endodontic bioactive cement MTA HP repair compared with ProRoot MTA white and NeoMTA plus. J. Biomed. Mater. Res. B Appl. Biomater. 2019, 107, 2109-2120. [CrossRef]

38. Jiménez-Sánchez, M.C.; Segura-Egea, J.J.; Díaz-Cuenca, A. A Microstructure Insight of MTA Repair HP of Rapid Setting Capacity and Bioactive Response. Materials 2020, 13, 1641. [CrossRef]

39. Jiménez-Sánchez, M.D.C.; Segura-Egea, J.J.; Díaz-Cuenca, A. MTA HP Repair stimulates in vitro an homogeneous calcium phosphate phase coating deposition. J. Clin. Exp. Dent. 2019, 11, e322-e326. [CrossRef]

40. Duarte, M.A.H.; Minotti, P.G.; Rodrigues, C.T.; Ordinola-Zapata, R.; Bramante, C.M.; Filho, M.T.; Vivan, R.R.; de Moraes, I.G.; de Andrade, F.B. Effect of Different Radiopacifying Agents on the Physicochemical Properties of White Portland Cement and White Mineral Trioxide Aggregate. J. Endod. 2012, 38, 394-397. [CrossRef]

41. Nekoofar, M.H.; Aseeley, Z.; Dummer, P.M.H. The effect of various mixing techniques on the surface microhardness of mineral trioxide aggregate. Int. Endod. J. 2010, 43, 312-320. [CrossRef]

42. Camilleri, J. Hydration mechanisms of mineral trioxide aggregate. Int. Endod. J. 2007, 40, 462-470. [CrossRef]

43. American Dental Association. ANSI/ADA Specification 57; Section 5.8; American Dental Association: Chicago, IL, USA, 2000.

44. Viapiana, R.; Guerreiro-Tanomaru, J.M.; Tanomaru-Filho, M.; Camilleri, J. Investigation of the Effect of Sealer Use on the Heat Generated at the External Root Surface during Root Canal Obturation Using Warm Vertical Compaction Technique with System B Heat Source. J. Endod. 2014, 40, 555-561. [CrossRef]

45. Bodanezi, A.; Carvalho, N.; Silva, D.; Bernardineli, N.; Bramante, C.; Garcia, R.B.; De Moraes, I.G. Immediate and delayed solubility of mineral trioxide aggregate and Portland cement. J. Appl. Oral Sci. 2008, 16, 127-131. [CrossRef] [PubMed]

46. Basturk, F.B.; Nekoofar, M.H.; Günday, M.; Dummer, P.M. Effect of Various Mixing and Placement Techniques on the Flexural Strength and Porosity of Mineral Trioxide Aggregate. J. Endod. 2013, 40, 441-445. [CrossRef] [PubMed]

47. Parirokh, M.; Torabinejad, M. Mineral Trioxide Aggregate: A Comprehensive Literature Review—Part I: Chemical, Physical, and Antibacterial Properties. J. Endod. 2010, 36, 16-27. [CrossRef]

48. Scelza, M.Z.; Da Silva, D.; Scelza, P.; De Noronha, F.; Barbosa, I.B.; Souza, E.; De Deus, G. Influence of a new push-out test method on the bond strength of three resin-based sealers. Int. Endod. J. 2014, 48, 801-806. [CrossRef] [PubMed]

49. Huffman, B.P.; Mai, S.; Pinna, L.; Weller, R.N.; Primus, C.M.; Gutmann, J.L.; Pashley, D.H.; Tay, F.R. Dislocation resistance of ProRoot Endo Sealer, a calcium silicate-based root canal sealer, from radicular dentine. Int. Endod. J. 2009, 42, 34-46. [CrossRef] [PubMed]

50. Yu, L.; Wei, M. Biomineralization of Collagen-Based Materials for Hard Tissue Repair. Int. J. Mol. Sci. 2021, 22, 944. [CrossRef]

51. Kokubo, T. Bioactive glass ceramics: Properties and applications. Biomaterials 1991, 12, 155-163. [CrossRef]

52. Hilton, T.J.; Ferracane, J.L.; Mancl, L. Northwest Practice-based Research Collaborative in Evidence-based Dentistry (NWP). Comparison of $\mathrm{CaOH}$ with MTA for direct pulp capping: A PBRN randomized clinical trial. J. Dent. Res. 2013, 92 (Suppl. 7), 16S-22S. [CrossRef] [PubMed] 
53. Gandolfi, M.G.; Siboni, F.; Botero, T.; Bossù, M.; Riccitiello, F.; Prati, C. Calcium Silicate and Calcium Hydroxide Materials for Pulp Capping: Biointeractivity, Porosity, Solubility and Bioactivity of Current Formulations. J. Appl. Biomater. Funct. Mater. 2015, 13, 43-60. [CrossRef] [PubMed]

54. Formosa, L.M.; Mallia, B.; Camilleri, J. The effect of curing conditions on the physical properties of tricalcium silicate cement for use as a dental biomaterial. Int. Endod. J. 2011, 45, 326-336. [CrossRef]

55. Gandolfi, M.G.; Taddei, P.; Tinti, A.; Dorigo, E.D.S.; Prati, C. Alpha-TCP improves the apatite-formation ability of calcium-silicate hydraulic cement soaked in phosphate solutions. Mater. Sci. Eng. C 2011, 31, 1412-1422. [CrossRef]

56. Van Meerloo, J.; Kaspers, G.J.L.; Cloos, J. Cell sensitivity assays: The MTT assay. Methods Mol. Biol. 2011, 731, 237-245. [CrossRef]

57. Camilleri, J. Staining Potential of Neo MTA Plus, MTA Plus, and Biodentine Used for Pulpotomy Procedures. J. Endod. 2015, 41, 1139-1145. [CrossRef] [PubMed]

58. Al-Sa'Eed, O.R.; Al-Hiyasat, A.S.; Darmani, H. The Effects of Six Root-end Filling Materials and Their Leachable Components on Cell Viability. J. Endod. 2008, 34, 1410-1414. [CrossRef]

59. Akbulut, M.B.; Arpaci, P.U.; Eldeniz, A.U. Effects of novel root repair materials on attachment and morphological behaviour of periodontal ligament fibroblasts: Scanning electron microscopy observation. Microsc. Res. Tech. 2016, 79, 1214-1221. [CrossRef] [PubMed]

60. Pires, C.W.; Botton, G.; Cadoná, F.C.; Machado, A.K.; Azzolin, V.F.; da Cruz, I.; Sagrillo, M.; Praetzel, J.R. Induction of cytotoxicity, oxidative stress and genotoxicity by root filling pastes used in primary teeth. Int. Endod. J. 2015, 49, 737-745. [CrossRef] 